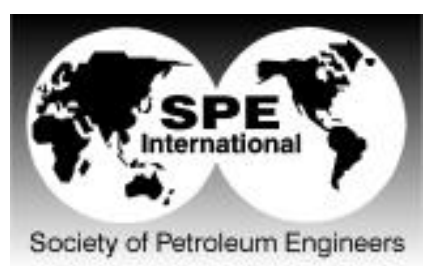

\title{
SPE 84439
}

\section{Using Visualization Tools to Gain Insight Into Your Data \\ C. Plaisant, G. Chintalapani, University of Maryland; C. Lukehart, D. Schiro and J. Ryan, ChevronTexaco}

Copyright 2003, Society of Petroleum Engineers Inc.

This paper was prepared for presentation at the SPE Annual Technical Conference and Exhibition held in Denver, Colorado, U.S.A., 5 - 8 October 2003.

This paper was selected for presentation by an SPE Program Committee following review of information contained in an abstract submitted by the author(s). Contents of the paper, as presented, have not been reviewed by the Society of Petroleum Engineers and are subject to correction by the author(s). The material, as presented, does not necessarily reflect any position of the Society of Petroleum Engineers, its officers, or members. Papers presented at SPE meetings are subject to publication review by Editorial Committees of the Society of Petroleum Engineers. Electronic reproduction, distribution, or storage of any part of this pape for commercial purposes without the written consent of the Society of Petroleum Engineers is prohibited. Permission to reproduce in print is restricted to an abstract of not more than 300 words; illustrations may not be copied. The abstract must contain conspicuous acknowledgment of where and by whom the paper was presented. Write Librarian, SPE, P.O. Box 833836, Richardson, TX 75083-3836, U.S.A., fax 01-972-952-9435.

\section{Abstract}

When the term 'visualization' is used in the oil and gas industry, it is usually referring to the viewing of complex geologic structures in three-dimensional space. This paper illustrates insights gained by applying interactive visual environments to petroleum industry data that has traditionally been presented in spreadsheets, line graphs, and bar charts. Two information visualization tools, Treemap and SpaceTree, will be shown applied to a variety of oilfield related data.

\section{Introduction}

This paper explores how Information Visualization tools can benefit the petroleum industry by providing novel interactive visual environments to explore data that has traditionally been presented in spreadsheets, line graphs, and bar charts.

Information visualization can be defined as the use of computer-supported interactive visual representation of abstract data to amplify cognition [1]. The abstract characteristic of the data is what distinguishes information visualization from scientific visualization. Information visualization is more likely to be used to display database content (e.g. recorded stock values, health statistics) than output of models or simulations. The display of georeferenced data is often a hybrid visualization that combines abstract and concrete data.

Information visualization aims to provide compact graphical presentations and user interfaces for interactively manipulating large numbers of items $\left(10^{2}-10^{6}\right)$, possibly extracted from far larger datasets [2, 3, 4.] Information visualization, sometimes called visual data mining, uses the remarkable human visual system to enable users to make discoveries or decisions, or propose explanations about patterns or exceptions. Perceptual psychologists and graphic designers (e.g Tufte, 1983) provide guidance about presenting static information, while the growing power of workers' desktop allows user-interface designers to propose rich interactions mechanisms to manipulate the data in real time.

The paper will now describe the results of the collaboration between a university research laboratory and a major oil company to investigate the application of two information visualization tools, Treemap and SpaceTree, to oilfield related data.

\section{Treemap}

Envision the task of managing the performance of a hundred compressors to minimize production downtime. Treemap aids in this process by providing an abstract visual representation of the compressors of interest in a single screen display that can be grouped and arranged by geographic region, asset team, field or platform. Each individual compressor is represented in the Treemap by a rectangular area whose characteristics are mapped to attributes of the compressors chosen by the engineer. For example the Treemap shown in Figure 1 represents 68 compressors grouped in a hierarchy of fields, asset teams, and regions. Each rectangle corresponds to a compressor, the size of the box is proportional to the flow rate, and color is a shade of red proportional to the number of abnormal compressor parameters. The engineer is now able to spot at a glance the compressors that need attention first (i.e. the compressors corresponding to large red rectangles).

The user interface provides many features to allow users to customize the display to their particular needs. Most importantly, users can specify what data attribute should be mapped to the size or color of the rectangles. For example users can map the size of the rectangle to the average number of monthly problems and map the color to the age of the compressor.

Interface features also help users focus on areas of interest or get more details about he compressors. A window popup shows the long labels that may not fit on the rectangle; clicking on a rectangle displays all the detailed information available about a compressor in the top right area of the display. Users can zoom on part of the Treemap, for example on a specific field, which is useful when the number of managed compressors becomes very large. They can also filter the display using dynamic query sliders or controls [5] to show only the compressors that have characteristics that fall 
within specified ranges. Examples of dynamic query sliders are shown at the lower right of Figure 1. As users adjust the position of the sliders, the rectangles that fall outside the range are grayed out dynamically (e.g. users can filter out the compressors with flow rates less than 20 cubic feet per minute). A click on the "Hide filtered" button removes the gray rectangles and gives more room to the remaining compressors.

Other example of use: Daily Production Report. Treemap has also been applied to the monitoring of daily production from oil and gas wells. The Treemap shown in Figure 2 summarizes the production of 691 wells grouped by Business Unit, Area, and then Asset Team. Each box in the Treemap represents a well. The size of the rectangle is proportional to number of barrels of oil produced per day. The color indicates "lost" production - the difference between the actual and expected barrels produced. The Treemap shows at a glance which of the wells are the largest producers (i.e. the large rectangles), and the color indicates which wells are not producing as expected.
Example of Use - Project Management Metrics Tracking. Another use for Treemap is to track the status and progress of capital projects. In this example the Treemap is dis playing information on 180 capital projects being worked on in an offshore Gulf of Mexico business unit (Figure 3). The projects in this display are grouped by field and then by department. The size of the rectangle represents the gross budget amount. Color is used to highlight which projects have run over budget by coloring them in red.

Treemap also allows users to change the hierarchy of projects interactively by changing the attributes used to group the data. For example, users can select the attribute "status" to separate the projects in the early stages of development from the ones in latter stages, and then select "owner" to group projects within those categories by the owner who manges the project.

In all of the examples mentioned above, an employee in charge of analyzing data and preparing reports can explore the various possible Treemap settings and then save the preferred settings for others to use. The data used to populate the Treemap can be imported from a database at regular intervals making up-to-date information easily available for monitoring.

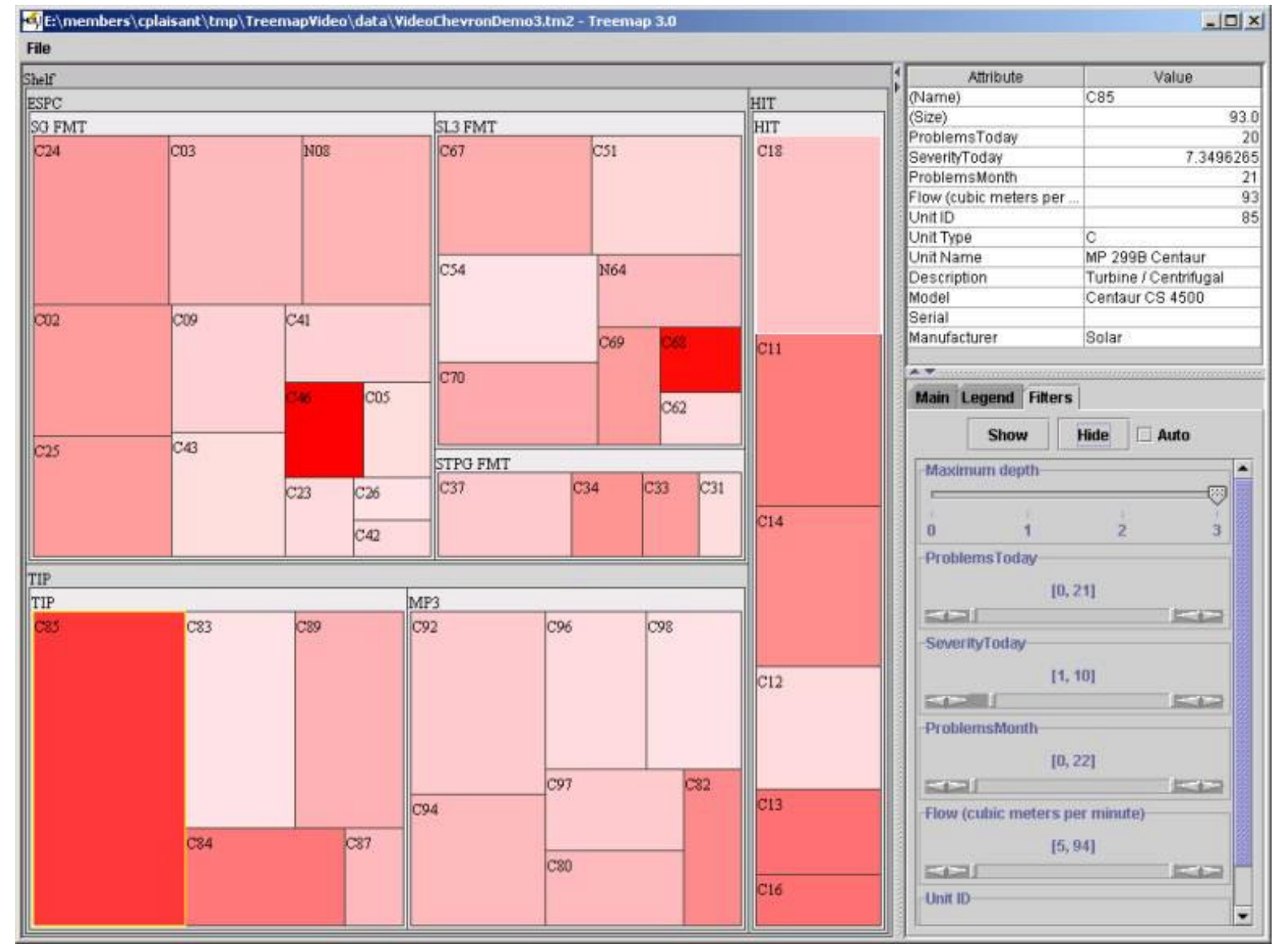

Figure 1: Sixty eight compressors grouped in a hierarchy of fields, asset teams, and regions. Each rectangle is a compressor, the size of the rectangle is proportional to the flow rate, and the shade of red is proportional to the number of abnormal compressor parameters exhibited. 


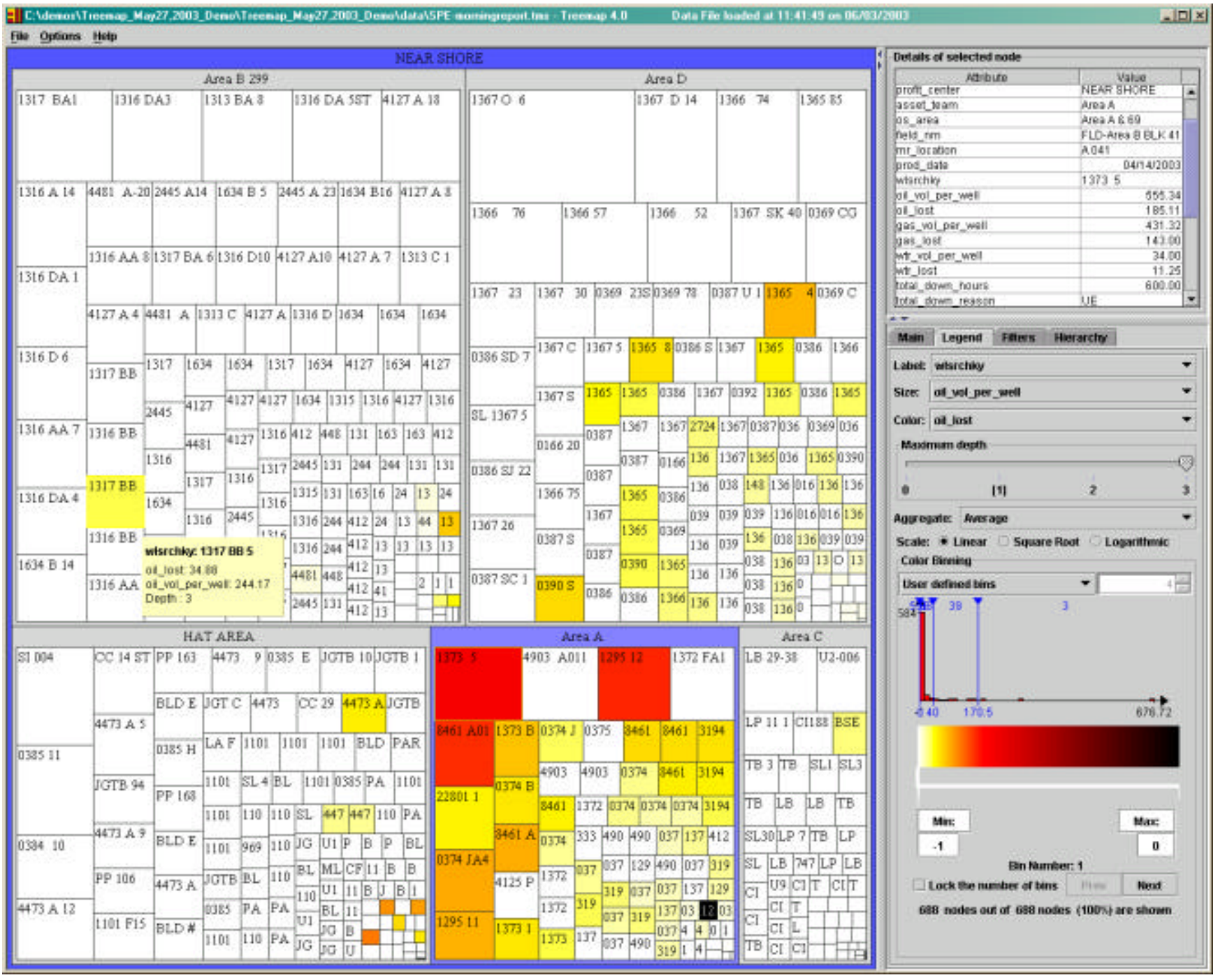

Figure 2: Each box represents a well. The 691 wells are grouped by Asset team. The size of the box is proportional to the barrels of oil produced per day, and the color shade indicates "lost" oil (difference between actual and expected), dark areas indicate problems.

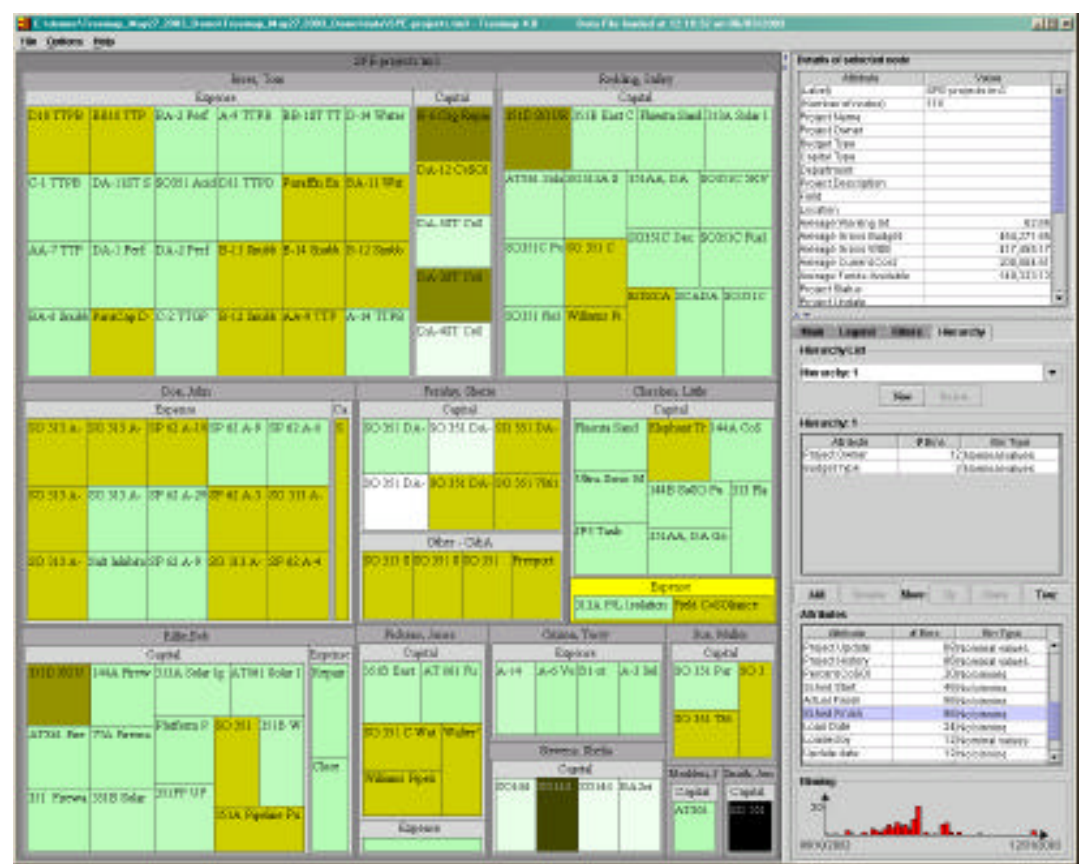

Figure 3: All of the projects managed by a business unit are summarized in one display. Each rectangle is a project. Thecolor of the rectangle indicate the amount of available funds. White to light green colors indicates that funds are still available, while brown/dark colors indicates projects are over budget. (Note that red and green would be more appropriate colors for budgets but we chose colors that would be readable when printed in black and white.) Size is not used here so all rectangles have a similar area. Using the hierarchy control panel, users can specify how they want to group the projects, here by owner/manager, then by budget type (capital or expense.) 


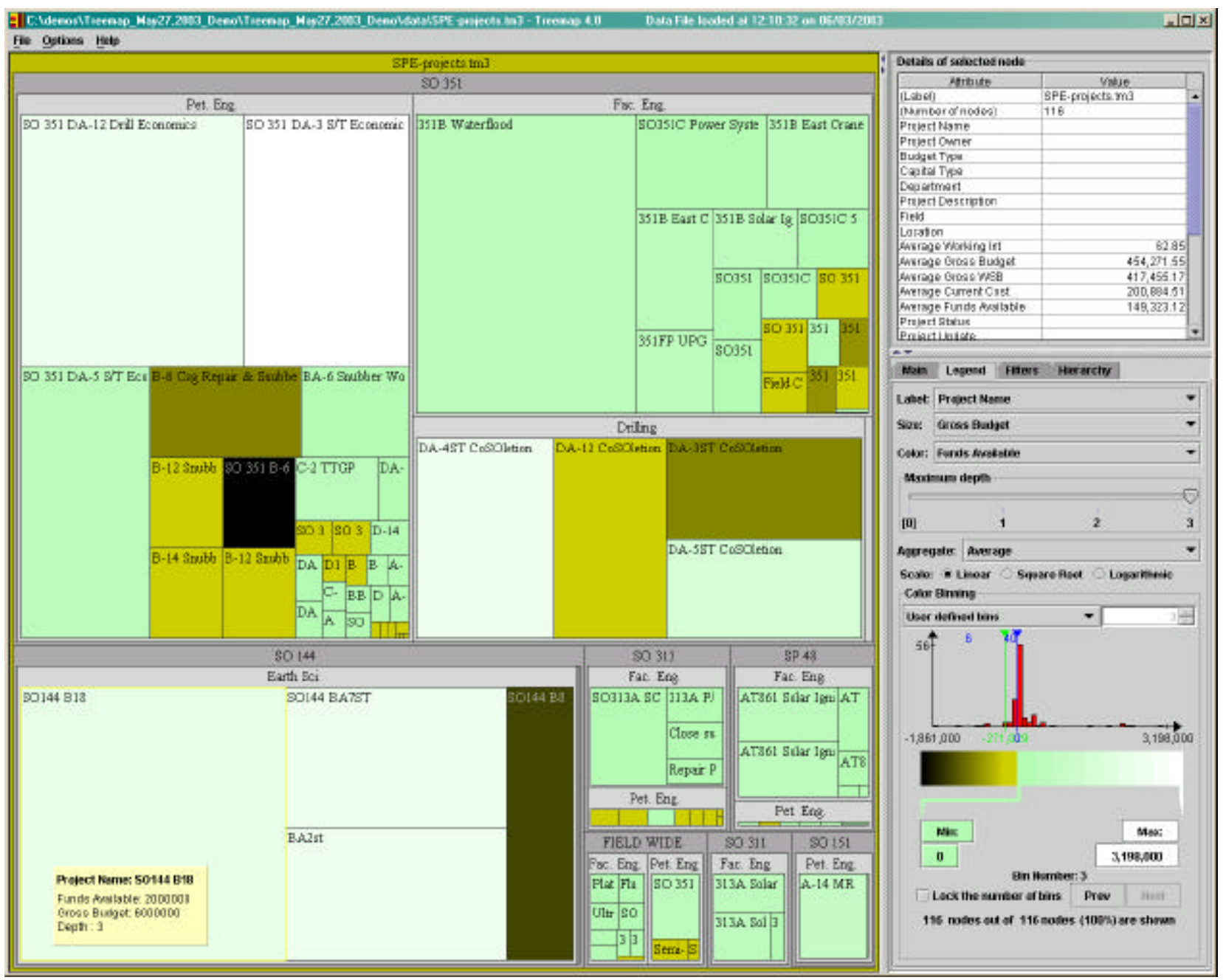

Figure 4: All of the projects managed by a business unit are summarized in a different display, this time grouped by field and department. The size of the rectangle represents the project budget, with dark colors indicating which projects are over budget.

The next section looks at the second information visualization tool we explored: SpaceTree.

\section{SpaceTree}

SpaceTree is designed to help a user visualize complex hierarchical relationships in data. One type of data that has traditionally been viewed in a "tree" type of format is an organizational chart (Figure 5). In large organizations these charts can be quite complex and are often broken up into a series of graphs that make the overall reporting relationships difficult to understand. SpaceTree allows for the interactive, dynamic rescaling of the branches of the tree to best fit the available screen space. Large trees can be explored dynamically. When users select a node to change the focus of the layout, the number of levels opened is maximized and the tree is animated to its new layout. Users can navigate the tree by clicking on nodes to open branches, or by using the arrow keys to navigate among siblings, ancestors and descendants.

Branches that do mot fit on the screen are summarized and previewed as a triangle. The visual attributes of the preview triangle can reflect attributes of the branches. For example the shading of the triangle can be made proportional to the total number of nodes in the subtree (e.g. the number of employees) and the height of the triangle can represents the depth of the subtree hierarchy.

Several SpaceTree layout options allow adjustments of the spacing between nodes, alignment, icon options etc. The choice of overall orientation of the tree layout, allows designers or users to match the layout to the natural orientation of the data. For example organizational charts are often oriented top down (suggesting power), while other trees might benefit from being drawn left to right (suggesting time) or bottom up (suggesting progress). The choice of the most space efficient orientation depends on the tree topology and the aspect ratios of the labels and the window.

SpaceTree also contains a search function to allow the user to zero in on the data item of interest. For example in Figure 6 the user has typed the word "scientist". Colored highlights now show where in the organization chart all the scientists are to be found. Hitting the "return" key lets the user "trim the tree" to only show the branches leading to the scientists (Figure 7). This facilitates reviewing the results of the search in the context of the hierarchical structure. 
Other examples of use. SpaceTree has also been used to help manage the regional IT support of numerous Business Divisions with a number of remote sites. Employees can learn the structure of the organization by reviewing up-to-date information online ( 8 and 9) and with that knowledge solve some problems faster. For example, imagine that the central IT helpdesk receives a support call from a small Marketing office in Baton Blanc, Louisiana. The closest Marketing office with onsite IT support with the knowledge to solve the problem is located in Dallas, Texas. By using the search capability in SpaceTree the helpdesk is able to quickly identify that the Pipeline Division also has an office in Baton Blanc with the needed support personnel (see figures 10 and 11). By leveraging support staff across Business Divisions, travel costs are reduced and the customer's problem is addressed much more quickly.

The SpaceTree can also be used as a tool for accessing documents in the context of the organization structure. In the regional IT support example, each Business Division requires a different mix of IT services to fit their particular business needs. This information can be made easily accessible to the Business Division or IT helpdesk personnel by attaching a copy of the different IT Service Agreements to each node (see example of figure 12.)

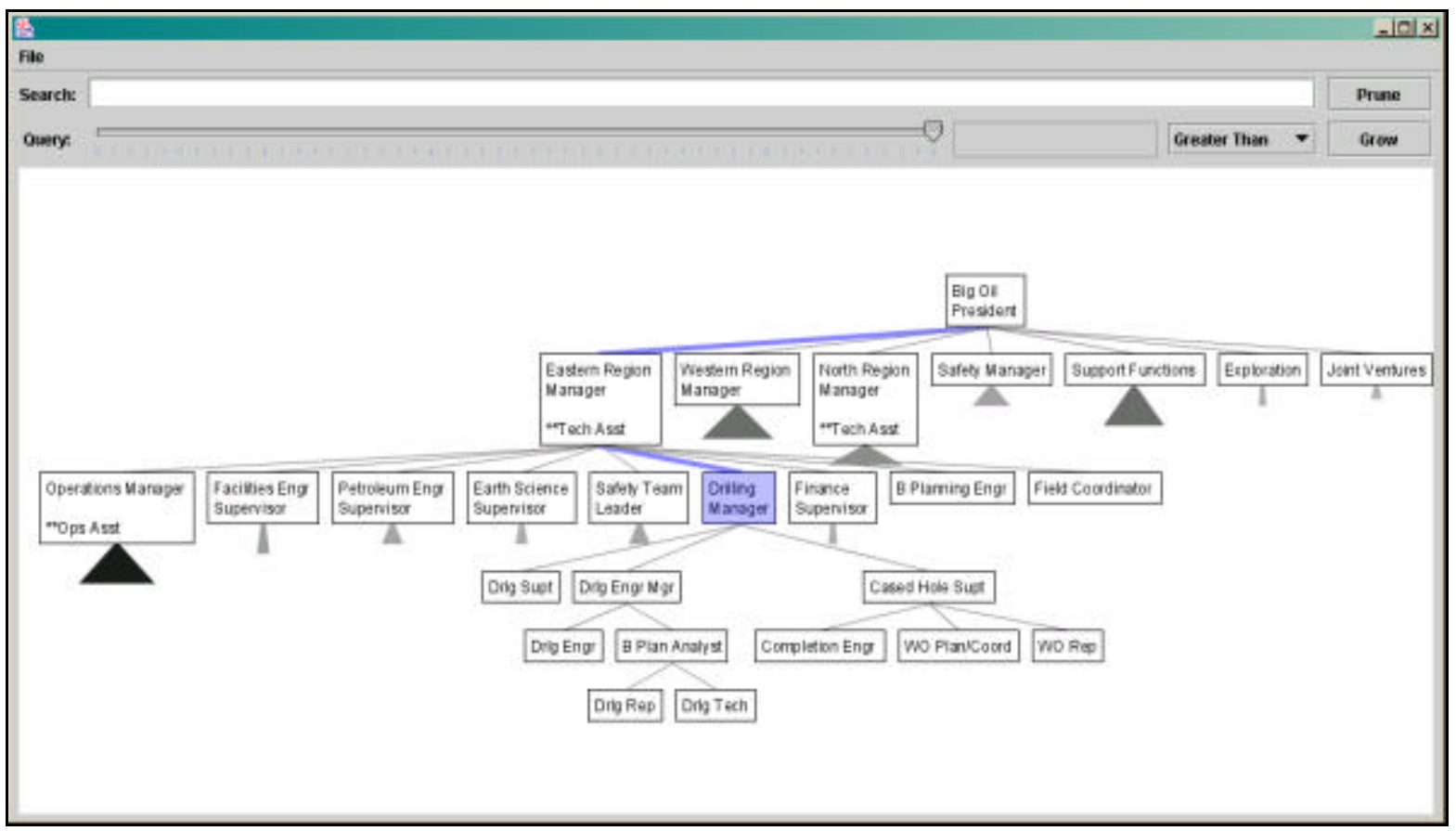

Figure 5: SpaceTree showing an organization chart. The 3 lower levels of the hierarchy were opened at once as users clicked on "Drilling Manager" (the colored node in the middle.) Smooth animated transitions allow users to remain oriented when the layout changes. 


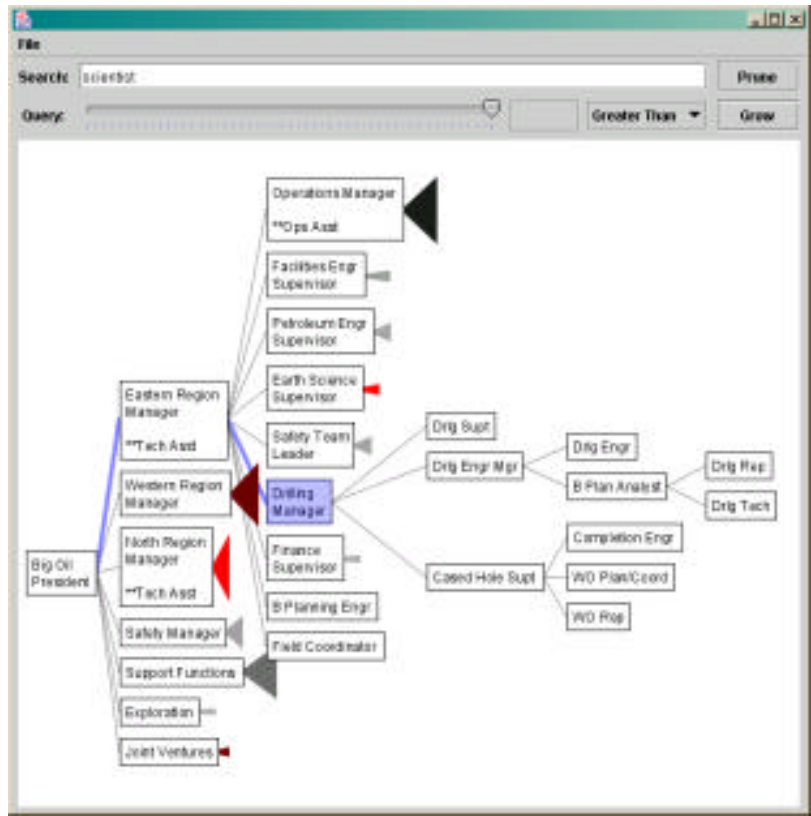

Figure 6: SpaceTree allows users to specify the orientation of the tree, for example here, setting the root toward the left. Here a search is underway, highlighting where in the tree the word "scientist" can be found.

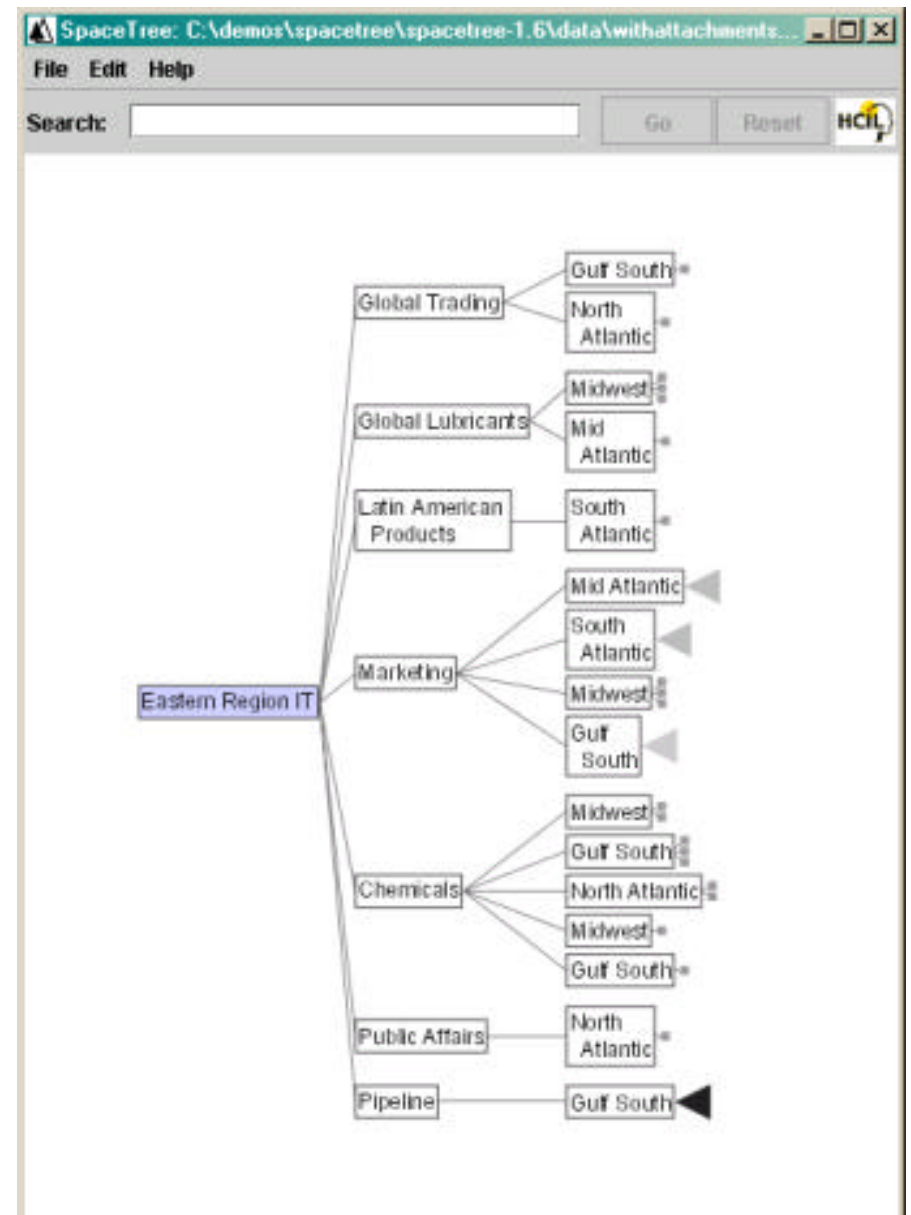

Figure 8: Another example of use showing a business division. The tree is opened to show as many nodes as will fit on the screen. Here two levels are opened.

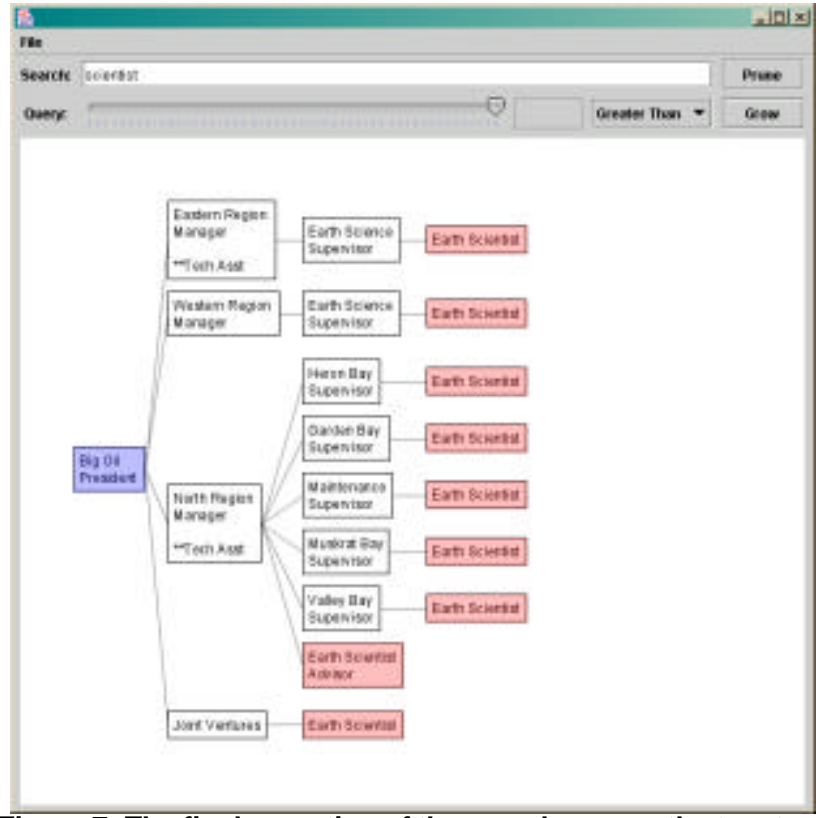

Figure 7: The final exeaution of the search prunes the tree to show only the branches that contain the result nodes, here all the scientists, in the context of their place in the organization

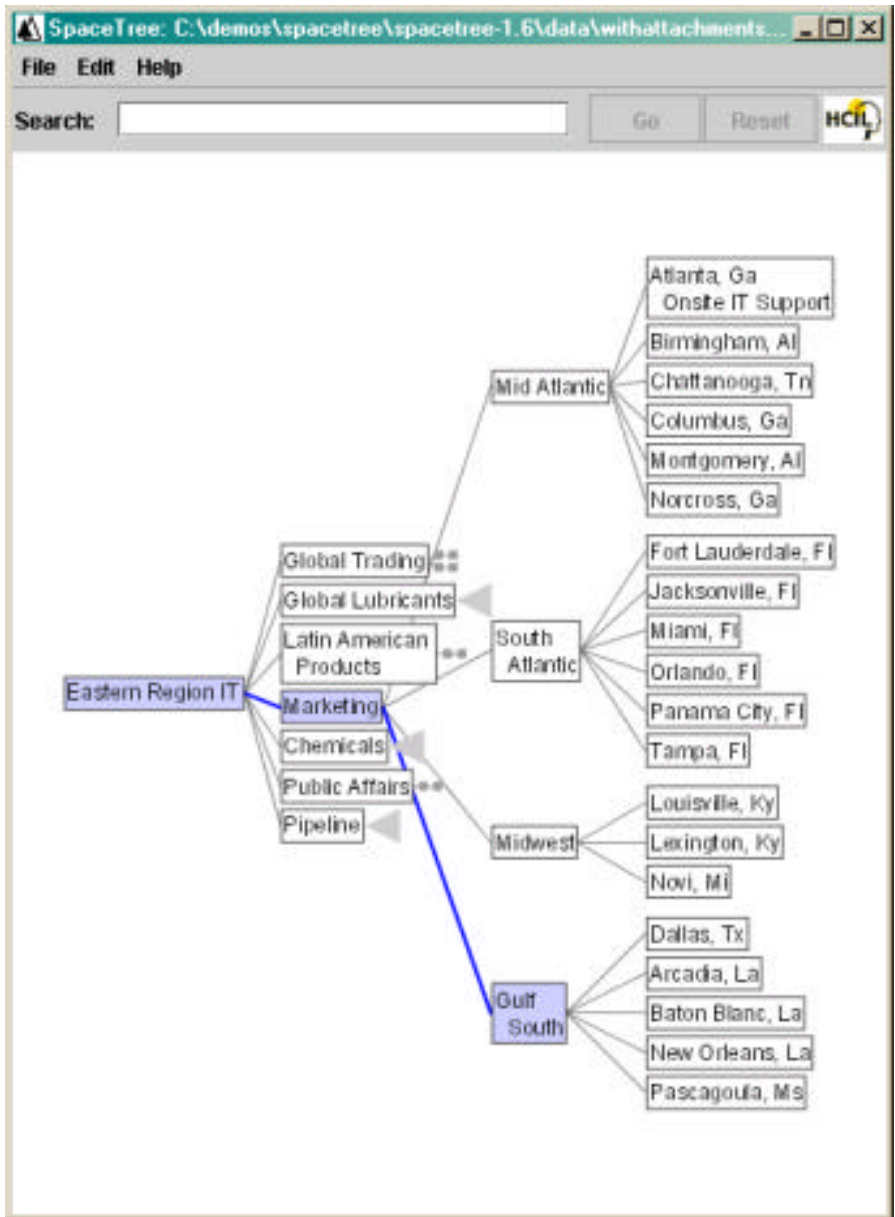

Figure 9: Now the user has clicked on "Gulf South" under Marketing. Higher level branches were closed while Gulf South was expanded to reveal its offices as well as the other Marketing offices. 


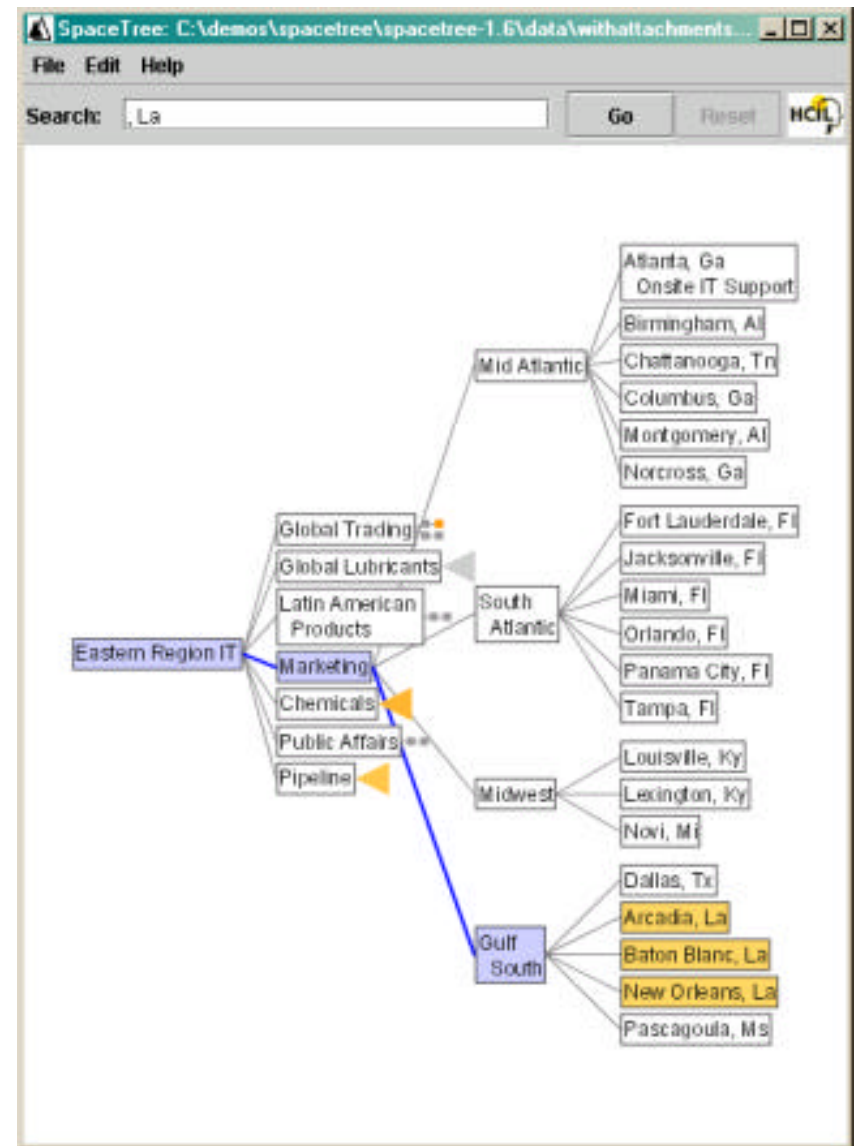

Figure 10: A search for all the Louisiana locations. As the user types ", La" all the nodes or branches containing that string are highlighted in yellow.

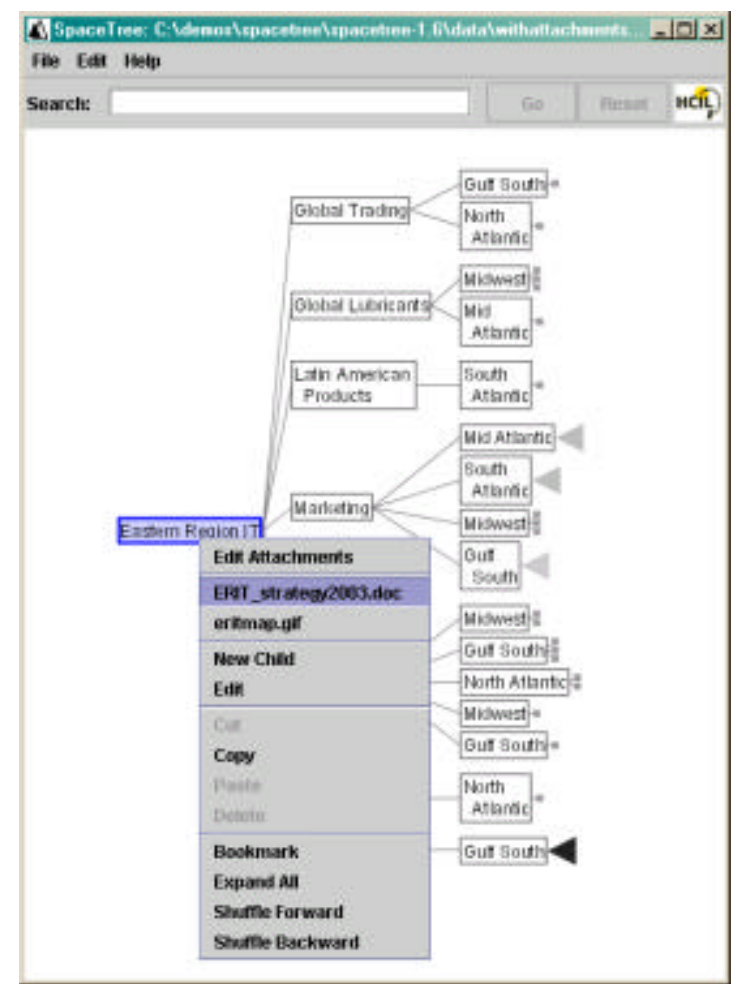

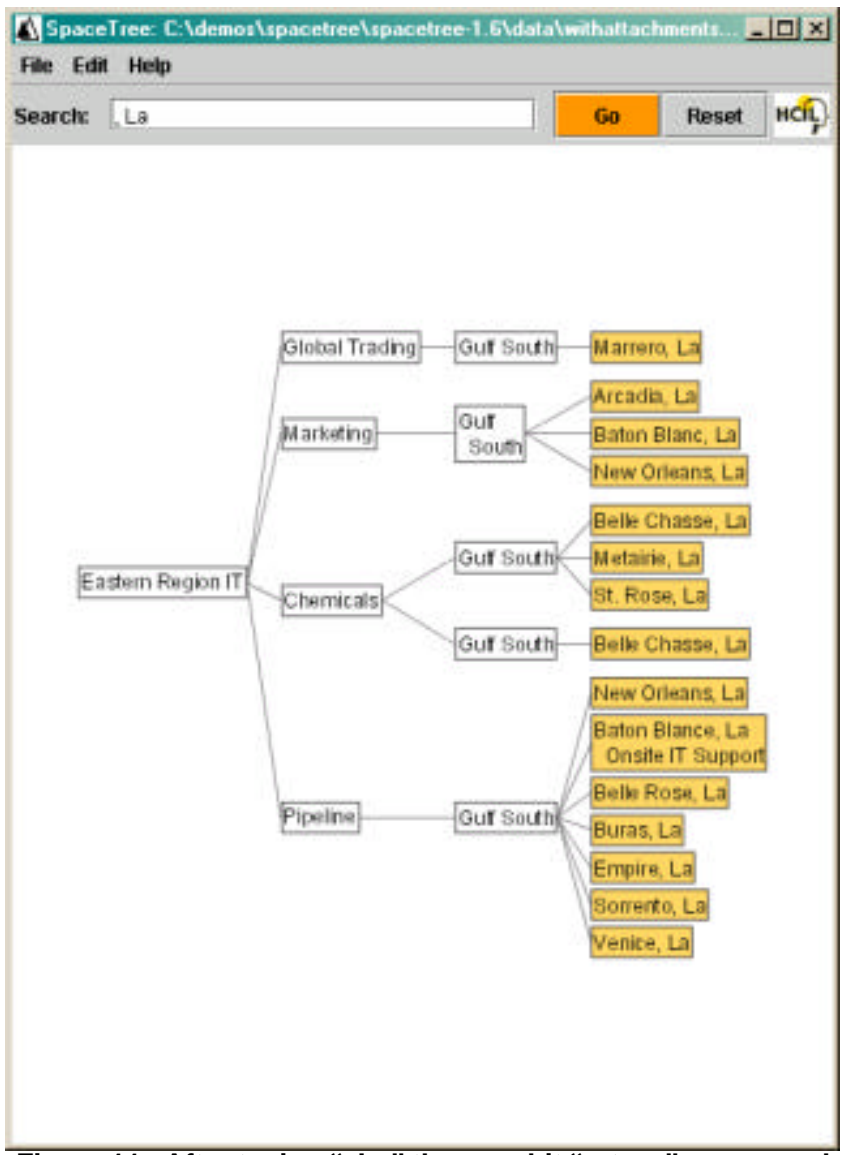

Figure 11: After typing ", La" the user hit "return" or pressed "go" and the tree is now pruned to only show the nodes containing the searched string, showing all the Louisiana offices in the context of the hierarchy.

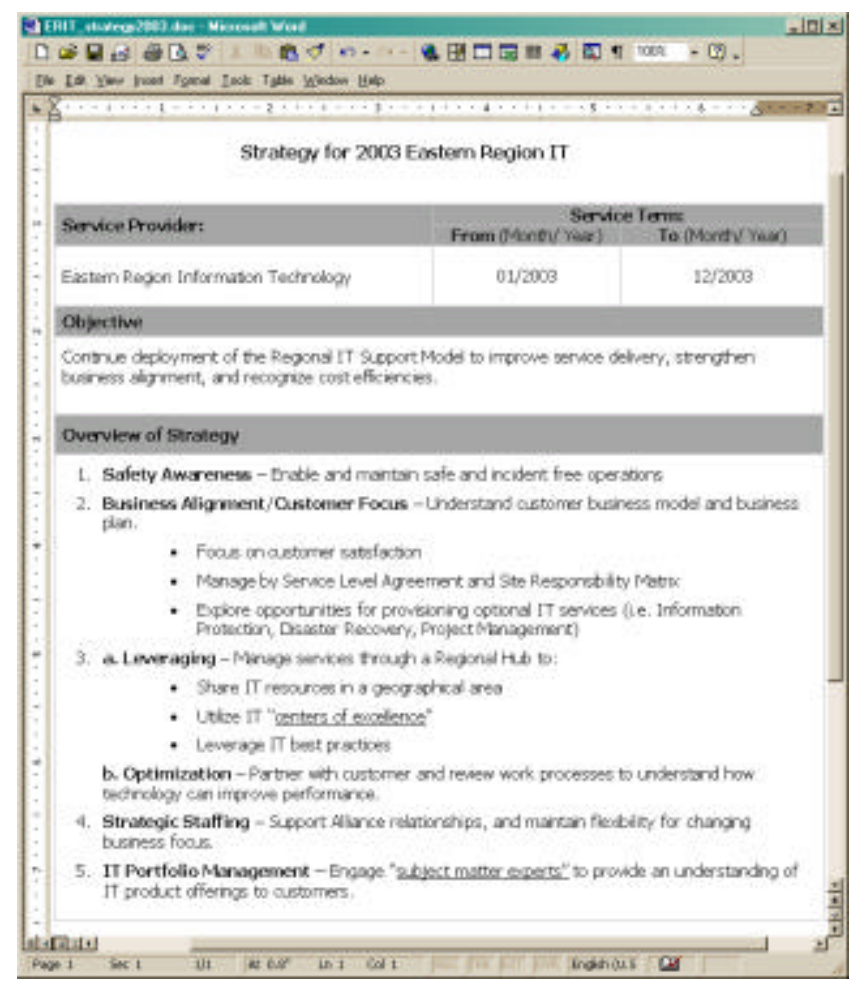

Figure 12: A right click on a node brings a menu that gives access to the documents associated with the node. When the document is selected, here a strategy document, it opens on a separate window. The menu also reveals some of the tree editing functions that are available only to users who would have editing privileges. 


\section{Background on Treemap and SpaceTree}

Both Treemap and SpaceTree have been developed at the University of Maryland Human-Computer Interaction Laboratory (www.cs.umd.edu/hcil.)

Treemap is a space-filling visualization method [6]. A treemap works by dividing the display area into a nested sequence of rectangles whose areas correspond to an attribute of the data set, effectively combining aspects of a Venn diagram and a pie chart. Originally designed to visualize files on a hard drive, treemaps have been applied to a wide variety of domains ranging from financial analysis ([7], see also http://www.smartmoney.com/marketmap) to sports reporting [8]. More recently alternative layout algorithms were devised to reduce the incidence of high aspect ratio rectangles and to maintain ordering [9]. Others have focused on specialized techniques to visualize up to a million items without aggregation [10.]

SpaceTree uses the more traditional representation of a node link tree diagrams. It adds dynamic rescaling of branches of the tree to best fit the available screen space, smooth animated transitions to maintain orientation, and the use of preview icons summarize the topology of the branches that cannot be to expanded [11].

Comparing the two techniques: Space filling techniques such as treemaps (or information slices [12]) have been successful at visualizing trees that have attributes values at the node level. Space filling techniques shine when users care mostly about leaf nodes and their attributes (e.g. outlier stocks or malfunctioning compressors) but do not need to focus on the topology of the tree, or the topology of the tree is trivial (e.g. 2 or 3 fixed levels). Treemap users require some training because of the unfamiliar layout.

Traditional node link diagrams, on the other hand, make inefficient use of screen space, leaving the root side of the tree completely empty - usually the top or left of the screen - and overcrowding the opposite side. Even trees of a hundred nodes often need multiple screens to be completely displayed, or require scrolling since only part of the diagram is visible at a given time. Specialized tools can help users manage the multiple pages needed to display those trees (e.g. www.nakisa.com for organizational chart). Optimized layout techniques can produce more compact displays by slightly shifting branches or nodes but those techniques only partially alleviate the problem and are often not appropriate for interactive applications. SpaceTree use of preview icons, smooth animated transitions and filtering alleviates some of those problems while remaining and easy to use and understand technique requiring no training.

Of course it is possible to combine the two techniques, either to introduce Treemap users to the unusual layout or to explore two independent hierarchies simultaneously [13.]
Related research: Many other approaches to node link tree visualization have been explored. SpaceTree shares many features with the Degree-of-Interest Tree developed at about the same time by Card and Nation [14]. Other approaches include the Cone Trees [15] which allow users to rotate a 3D representation of the tree to reveal its hidden parts, but occlusion and the additional complexity navigating the tree or keeping oriented can be a problem. A clever way to make better use of screen space is to break loose from the traditional up-down or left-right orientation and use circular layouts. The best known technique is the Hyperbolic tree browser [16] now available as StarTree - which uses hyperbolic geometry to place nodes around the root and provides smooth and continuous animation of the tree as users click or drag nodes to readjust the focus point of the layout. The animation is striking but the constant redrawing of the tree can be distracting. Labels are hard to browse because they are not aligned and sometimes overlap. In addition, the unconventional layout may not match the expectations of users (e.g. it is not appropriate to present the organizational chart of a conventional business.)

\section{Conclusions}

Our exploratory work provides insight into how information visualization can be used to support discovery and problem solving during the examination of petroleum industry data. To help users cope with vast datasets, designers have improved on 250-year old visual designs such as histograms and plots that work best with only a few dozen data points. Information visualization provides new strategies for making sense of abstract information spaces and user interface controls for coordinating them. As users move sliders, zoom in on aggregates, and make selections, they can follow changing patterns and find problems. While a picture may be worth a thousand words, an interface is worth a thousand pictures.

\section{Acknowledgement}

We want to thank Tommy Mertz, Charlie Guthrie, Bill Kowalik and Trond Unneland for their support in the development of the oil industry applications, Ben Shneiderman, Niem Dang and Ani Jain for their collaboration in the latest developments of Treemap, and Jesse Grosjean and Ben Bederson for their collaboration in the design and development of SpaceTree.

\section{References}

1. Card, S., Mackinlay, J., and Shneiderman, B., Readings in Information Visualization: Using Vision to Think, Morgan Kaufmann Publ., San Francisco, CA (1999)

2. Spence, Robert, Information Visualization, AddisonWesley, Essex, England (2001).

3. Bederson B., Shneiderman, B., The Craft of Information Visualization: Readings and Reflections, Morgan Kaufman, 2003 
4. Tufte, Edward, The Visual Display of Quantitative Information, Graphics Press, Cheshire, CT (1983).

5. Ahlberg, Christopher, Williamson, Christopher, and Shneiderman, Ben, Dynamic queries for information exploration: An implementation and evaluation, Proc. ACM CHI '92: Human Factors in Computing Systems, ACM, New York (1992), 619-626.

6. Shneiderman, Ben, Tree visualization with tree-maps: A 2D space-filling approach, ACM Transactions on Graphics, 11, 1 (January 1992), 92-99.

7. Wattenberg, M. Map of the Market (1998). Wattenberg, M. (1999). Visualizing the Stock Market. In Proceedings of Extended Abstracts of Human Factors in Computing Systems (CHI 99) ACM Press, New York, pp. 188-189.

8. Jin, L., \& Banks, D. C. (1997). TennisViewer: A Browser for Competition Trees. IEEE Computer Graphics and Applications, 17(4), pp. 63-65.

9. Bederson, B.B., Shneiderman, B., and Wattenberg, M. Ordered and Quantum Treemaps: Making Effective Use of 2D Space to Display Hierarchies, ACM Transactions on Graphics (TOG), 21, (4), October 2002, 833-854

10. Fekete, J. and Plaisant, C., Interactive Information Visualization of a Million Items, proc. of IEEE conference on Information Visualization, Boston, Sept. 2002 (2002) 117-124

11. Plaisant, C., Grosjean, J., Bederson, B., SpaceTree: Supporting Exploration in Large Node Link Tree, Design Evolution and Empirical Evaluation, proc. of IEEE conference on Information Visualization, Boston, Sept. 2002 (2002) 57-64
12. Andrews, K., Heidegger, H. (1998) Information Slices: Visualising and exploring large hierarchies using cascading, semicircular disks. Proc of IEEE Infovis'98 late breaking Hot Topics IEEE, 9-11. ftp://ftp.iicm.edu/pub/papers/ivis98.pdf

13. Kules, W., Shneiderman, B., and Plaisant, C. (2003). Data Exploration with Paired Hierarchical Visualizations: Initial Designs of PairTrees. To appear in Proc. 2003 National Conference on Digital Government Research. http://www.dgrc.org/dgo2003

14. Card, S., Nation, D., Degree-of-Interest Trees: a component of attention-reactive user interface. Proc. of Advanced Visual Interface '02, May 22-24, 2002., ACM, New York

15. Robertson, G. G. Mackinlay, J. D. Card, S. K. Cone Trees: animated 3D visualizations of hierarchical information, Proc. Human factors in computing systems conference, March 1991, 189-194

16. Lamping, J., Rao, R., Pirolli; P. (1995) A focus+context technique based on hyperbolic geometry for visualizing large hierarchies Conference proceedings on Human factors in computing systems, 1995, 401-408

\section{Additional resources:}

For more information and download demonstrations of Treemap see: http://www.cs.umd.edu/hcil/treemap3

For more information and download demonstrations of SpaceTree see: http://www.cs.umd.edu/hcil/spacetree 